\title{
EFFICIENCY AND CHOQUET BOUNDARIES IN SEPARATED LOCALLY CONVEX SPACES
}

\author{
VASILE POSTOLICĂ
}

Received 10 March 2002

\begin{abstract}
This research paper is devoted to the study of the properties for Pareto-type efficient point sets in separated locally convex spaces, based upon an earlier result on the coincidence of Pareto-type efficient point sets and the Choquet boundaries and the natural corresponding extension for the approximate efficient point sets in Hausdorff locally convex spaces. Both of these results represent an important connection between two great fields of mathematics: vector optimization and potential theory.
\end{abstract}

2000 Mathematics Subject Classification: 90C29, 90C48.

1. Introduction. The significant result set forth in [7] is thought not to be sufficiently well known even if one colligates the coincidence between the set of all Pareto-type minimum points of any nonempty, compact set, with respect to the order relation given by an arbitrary closed, convex, pointed cone in every Hausdorff locally convex space and the Choquet boundary of the same set with respect to a convenient convex cone of real continuous functions defined on the set, all of these functions increasing in comparison with the order relation induced by the cone. The natural "mutatis mutandis" generalization for the efficiency regarding the Pareto-type maximum points sets, together with the immediate consequences, some important open problems coupled with the recent investigations of the efficient points, and the main properties for the efficient points sets in the best appropriate framework offered by the separated locally convex spaces ordered by the supernormal cones introduced by Isac in 1981 and published in 1983 [8] (see also, for instance, [9, 10, 11, 12, 17, 18] and all the other connected research works) suggest a new expectation for the possible reassessment of the Choquet boundaries and the corresponding extensions. Simultaneously, the natural generalization of the pertinent coincidence result given in [7] for the approximate efficient point sets obtained by us in recent scientific studies and based on the properties of Pareto-kind sets [18] and other related research papers (see, e.g., [13, 15, 17, 19], etc.) made us determined to continue the study of the properties for the efficient point sets through the agency of the Choquet boundaries and conversely. Thus, Section 2 is dedicated to the Choquet boundaries; it presents some important links between this subject and Altomare projections and relevant examples on the Choquet boundaries. Section 3 deals with the coincidence results and related topics. In Section 4 we formulate a few suitable open problems. All the elements of ordered topological vector spaces used in this work are in accordance with [16]. 
2. Choquet boundaries, interesting examples, and connections with Altomare projections. Taking into account the significance of the subsequent theorems in Section 3 in which we establish a very important relationship between vector optimization and the potential theory, together with their applications, we initially offer the theoretical details, the suitable contents, the adequate proofs, important examples, and some connections with Altomare projections. We continue the study of the efficient point sets through the agency of the Choquet boundaries in Section 3. Thus, we consider an arbitrary Hausdorff locally convex space $(E, \tau)$ where $\tau$ denotes its topology, and let $K$ be a closed, convex, pointed cone in this background. On the vector space $E$ we consider the usual order relation $\leq_{K}$ associated with $K$ as follows: for $x, y \in E$ one defines $x \leq_{K} y$ if there exists $k \in K$ with $y=x+k$. Clearly, this order relation on $E$ is closed, that is, the set $G_{K}$ given by $G_{K}=\left\{(x, y) \in E \times E: x \leq_{K} y\right\}$ is a closed subset of $E \times E$ endowed with the product topology. If $X$ is now every nonempty compact subset of $E$, then the hypotheses concerning $K$ ensure the existence of the efficient points in $X$ (see, e.g., [21]). Following the main considerations and references given in [6], for every nonempty and compact subset $X$ of $E$, we recall same basic concepts and results in potential theory concerning the Choquet boundary of $X$ with respect to any convex cone of lower semicontinuous and lower-bounded real functions defined on $X$. Thus, we recall that if $S$ is a convex cone such as this satisfying the properties:

(a) for all $x \in X$, there exists $s \in S, s>0$ and $s(x)<+\infty$;

(b) $S$ separates linearly $X_{1}=\{x \in X: \exists s \in S$ with $s(x)<0\}$, that is, for every $x, y \in X_{1}, x \neq y$, there exist $s, t \in S$ finite in $x$ and $y$ such that $s(x) t(y) \neq$ $s(y) t(x)$, then on the set $M_{+}(X)$ of all positive Radon measures defined on $X$ one associates the following preorder relation: if $\mu, v \in M_{+}(X)$, then $\mu \leq_{S} v$ whenever $\mu(s) \leq v(s)$ for all $s \in S$.

Let $S_{1}$ be the convex cone of all lower semicontinuous and lower bounded real functions $s$ on $X$ having the following property: $x \in X$ and $\mu \leq_{S} \varepsilon_{x}$ with $\varepsilon_{x}(f)=f(x)$ for every real-valued, continuous function $f$ on $X$ being the Dirac measure implies that $\mu(s) \leq s(x)$. Any nonempty subset $T \subseteq X$ will be called $S$-boundary if whenever $s \in S_{1}$ and $s_{/ T} \geq 0$ it follows that $s \geq 0$. The small closed $S$-boundary is usually called the Silov boundary of $X$ with respect to $S$. A closed set $A \subseteq X$ is called $S$-absorbent if $x \in A$ and $\mu \leq_{S} \varepsilon_{x}$ implies that $\mu(X \backslash A)=0$. The set $\partial_{S} X=\left\{x \in X_{1}\right.$ : $\{x\}$ is $S$-absorbent $\}$ is named the Choquet boundary of $X$ with respect to $S$. The trace on $\partial_{S} X$ of the topology on $X$ in which the closed sets coincide with $X$ or with any of the $S$-absorbent subsets of $X$ contained in $X_{1}$ is usually called the Choquet topology of $\partial_{S} X$.

THEOREM 2.1. $\partial_{S} X$ is the small $S$-boundary subset of $X$ with respect to the inclusion relation which is nonempty if and only if $X_{1} \neq \varnothing$.

Proof. Since it is clear that $T \subseteq X$ is $S$-boundary if and only if $A \cap T \neq \varnothing$ for every nonempty and $S$-absorbent set $A \subseteq X_{1}$, it suffices to consider $\partial_{S} X$. Indeed, let $A$ be an arbitrary nonempty and $S$-absorbent subset of $X_{1}$. Then, because the class of all nonempty $S$-absorbent subsets of $A$ is inductively ordered with respect to the inclusion, there exists a minimal $S$-absorbent set $A_{0} \subseteq A$. But $S$ separates linearly $X_{1}$. Therefore, 
$A_{0}$ is a singleton set and obviously $A_{0} \cap \partial_{S} X \neq \varnothing$. Moreover, if $T$ is an arbitrary $S$ boundary and $x \in \partial_{S} X$, then $\{x\} \cap T \neq \varnothing$, that is, $x \in T$ and the proof is completed.

COROllary 2.2 (the minimum principle). (i) $s_{/ \partial_{S} X} \geq 0$ implies $s \geq 0$;

(ii) $s_{/ \partial_{S} X}>0$ implies $s_{/ X_{1}}>0$ for every $s \in S_{1}$;

(iii) the Silov boundary of $X$ with respect to $S$ coincides with the adherence of the Choquet boundary;

(iv) if $A$ is a nonempty $S$-absorbent subset of $X$ and one considers the convex cone $S_{A}=\left\{s_{/ A}: s \in S\right\}$, then $\partial_{S_{A}} A=A \cap \partial_{S} X$

(v) $x \in \partial_{S} X$ if and only if the Dirac measure $\varepsilon_{X}$ is minimal with respect to $S_{1}$; if, in addition, $\inf (s, 0) \in S$ whenever $s \in S$, then $x \in \partial_{S} X$ if and only if $\varepsilon_{x}$ is minimal with respect to $S$.

REMARK 2.3. In particular, if $S$ is a convex cone of real continuous functions on $X$ and we denote by $C(X)$ the usual Banach space of all real-valued, continuous functions on $X$, then a measure $\mu \in M_{+}(X)$ is minimal with respect to the previous preorder relation when $\mu\left(Q_{s} f\right)=\mu(f)$, for all $f \in C(X)$, where $Q_{s} f=\inf \{s \in S: f \leq s\}$. Hence, if $x \in X$, then the Dirac measure $\varepsilon_{x}$ is minimal if and only if $\varepsilon_{x}\left(Q_{S} f\right)=\varepsilon_{x}(f)$, that is, $Q_{S} f(x)=f(x)$, for all $f \in C(X)$, and in accordance with the above corollary we have $\partial_{S} X=\left\{x \in X: Q_{S} f(x)=f(x), \forall f \in C(X)\right\}$.

THEOREM 2.4. If, for every upper semicontinuous and upper-bounded real function $f$ on $X$, the function $Q_{S} f$ is upper semicontinuous on $\partial_{S} X$, in particular, if $S$ is an arbitrary convex cone of real-valued continuous functions, then $\partial_{S} X$ is a Baire subset of $X$ endowed with the trace topology.

Proof. Clearly, $Q_{S} f(x)=f(x)$ for every $x \in \partial_{S} X$ and any upper semicontinuous and upper-bounded real function $f$ on $X$. Let $\left(G_{n}\right)$ be a decreasing sequence of open sets in $X$ such that $G_{n} \cap \partial_{S} X(n \in \mathbb{N})$ is dense in $\partial_{S} X$. We will show that $\left(\bigcap_{n \in \mathbb{N}} G_{n}\right) \cap \partial_{S} X$ is also dense in $\partial_{S} X$. Indeed, if $G$ is an arbitrary open set such that $G \cap \partial_{S} X \neq \varnothing$, then, by considering the function $\varphi: X \rightarrow\{-1,1\}$ defined as

$$
\varphi(x)= \begin{cases}-1, & x \in G, \\ 1, & x \in X / G,\end{cases}
$$

one obtains $Q_{S} \varphi(x)=\varphi(x)=-1$, for all $x \in G \cap \partial_{S} X, Q_{S} \varphi(x) \geq 1$, for all $x \in X \backslash G$, and the set $A_{0}=\left\{x \in X: Q_{S} \varphi(x)<-1 / 2\right\}$ is a neighborhood for $G \cap \partial_{S} X$ with $A_{0} \subseteq G \cap X_{1}$. Therefore, there exists an open set $G_{0}$ so that $G_{0} \cap \partial_{S} X \neq \varnothing$ and $\bar{G}_{0} \subseteq A_{0}$, where $\bar{G}_{0}$ denotes its adherence. Let $\psi_{1}$ be the characteristic function of $X \backslash\left(G_{0} \cap G_{1}\right)$ and let $\psi_{n+1}$ be the characteristic function for $X \backslash\left[\operatorname{int}\left(K_{n}\right) \cap G_{n+1}\right]$. Since the function $u_{n}=$ $Q_{S} \psi_{n}$ is upper semicontinuous on $\partial_{S} X$ and $u_{n+1}(x)=Q_{S} \psi_{n+1}(x)=\psi_{n+1}(x)=0$ for every $x \in \operatorname{int}\left(K_{n}\right) \cap G_{n+1} \cap \partial_{S} X$, it follows that the set $\left\{x \in X: u_{n+1}(x)<1 / 2^{n+1}\right\}$ is a neighborhood for $\operatorname{int}\left(K_{n}\right) \cap G_{n+1} \cap \partial_{S} X$. Therefore, there exists a compact

$$
K_{n+1} \subset\left\{x \in X: u_{n+1}(x)<\frac{1}{2^{n+1}}\right\} \subset \operatorname{int}\left(K_{n}\right) \cap G_{n+1}, \quad \operatorname{int}\left(K_{n+1}\right) \cap \partial_{S} X \neq \varnothing, \quad n \in \mathbb{N} .
$$


Because $\operatorname{int}\left(K_{n+1}\right) \cap G_{n+2} \subseteq \operatorname{int}\left(K_{n}\right) \cap G_{n+1}$ for every $n \in \mathbb{N}$, we have $u_{n+1} \leq u_{n+2}$, for all $n \in \mathbb{N}$.

Let now $u=\sup _{n \in \mathbb{N}} u_{n}$ and $K=\bigcap_{n \in \mathbb{N}} K_{n}$. It is clear that

$$
K=\{x \in X: u(x)=0\} \neq \varnothing, \quad \forall x \in X, \quad u(x) \geq 1, \quad \forall x \in X / K .
$$

Moreover, $\mu\left(u_{n}\right) \leq u_{n}(x)$ and $\mu(u) \leq u(x)$ if $x \in X$ and

$$
\mu \in\left\{v \in M_{+}(X): v \leq_{S} \varepsilon_{x}\right\} .
$$

On the other hand,

$$
\mu(X / K) \leq \mu(u) \leq u(x)=0, \quad \forall x \in K, \mu \in\left\{v \in M_{+}(X): v \leq_{S} \varepsilon_{x}\right\}
$$

show that $K$ is an $S$-absorbent set in $X$ and $K \subseteq G_{0} \subseteq X$ implies that $K \cap \partial_{S} X \neq \varnothing$. Hence,

$$
K \cap \partial_{S} X \subseteq \bigcap_{n \in \mathbb{N}}\left(G_{n} \cap G_{0}\right) \cap \partial_{S} X \subseteq G \cap\left(\bigcap_{n \in \mathbb{N}} G_{n}\right) \cap \partial_{S} X,
$$

and the proof is complete.

DEFINITION 2.5. Any real function $s$ on $X$ is called strictly $S$-concave in $x \in X$ if it has the following properties:

(i) $\mu(s) \leq s(x)$ whenever $\mu \leq_{S} \varepsilon_{x}$;

(ii) if $\mu \leq_{S} \varepsilon_{x}$ and $\mu(s)=s(x)$, then $\mu=\varepsilon_{x}$.

THEOREM 2.6. If there exists at least one lower semicontinuous function $s$, lower bounded and strictly $S$-concave for any $x \in X_{1}$, then

$$
\partial_{S} X=\left\{x \in X_{1}: Q_{S}(-s)(x)=-s(x)\right\}
$$

Whenever $X$ is metrizable and $S$ represents any convex cone of real continuous functions defined on $X$, then the set of real, continuous, and strictly $S$-concave function in every $x \in X_{1}$ is nonempty. In all these cases, $\partial_{S} X$ is a $G_{\delta}$-set.

Proof. If $x \in X_{1}$ and $Q_{S}(-s)(x)=-s(x)$, then $\mu \leq_{S} \varepsilon_{x}$ implies that $\mu(-s) \leq$ $Q_{s}(-s)(x)=-s(x)$. Hence $s(x) \leq \mu(s)$ and because $\mu \leq_{s} \varepsilon_{x}$ it follows that $\mu(s)=s(x)$, that is, $\mu=\varepsilon_{x}$. Let now $X$ be metrizable and $S$ a convex cone of real continuous functions on $X$. If as above one considers again $C(X)$ being the usual Banach space of all real continuous functions on $X$ equipped with the topology induced by the supremum norm $\|\cdot\|$, then there exist a countable set $A=\left\{s_{n}: n \in \mathbb{N}\right\} \subset S_{1} \cap C(X)$ and the function $s: X \rightarrow \mathbb{R}$ defined by $s=\sum_{n \in \mathbb{N}}\left(1 / 2^{n}\right) \cdot\left(s_{n} /\left\|s_{n}\right\|\right)$ is strictly $S$-concave on $X$.

Since $\partial_{S} X=\left\{x \in X_{1}: Q_{S}(-s)(x)=-s(x)\right\}$ and the function $Q(-s)$ is upper semicontinuous, one concludes that

$$
\partial_{S} X=X_{1} \cap \bigcap_{n=1}^{\infty}\left\{x \in X: Q_{S}(-s)(x)<-s(x)+\frac{1}{n}\right\}
$$

as claimed. 
THEOREM 2.7. $\partial_{S} X$ is a compact topological space with respect to Choquet's topology.

Proof. This result is straightforward since the family of all nonempty $S$-absorbent subsets of $X_{1}$ is closed with respect to the intersection of the decreasing nets containing sets such as these.

THEOREM 2.8. The set $\partial_{S} X \cap\{x \in X: s(x) \leq 0\}$ is compact with respect to Choquet's topology for each $s \in S$.

Proof. Let $\left(A_{\alpha}\right)_{\alpha \in I}$ be any decreasing net of nonempty $S$-absorbent subsets of $X$ such that $A_{\alpha} \cap \partial_{S} X \cap\{x \in X: s(x) \leq 0\} \neq \varnothing$ with $s \in S$ arbitrary.

Since the set $\{x \in X: s(x) \leq 0\}$ is closed, it follows that

$$
K=\bigcap_{\alpha \in I} A_{\alpha} \cap\{x \in X: s(x) \leq 0\} \neq \varnothing .
$$

If one assumes that $K \cap \partial_{S} X=\varnothing$, then $K \cap \partial_{S_{A}}=\varnothing$ where $S_{A}=\left\{s_{/ A}: s \in S\right\}$. Consequently, $s(x)>0$, for all $s \in \partial_{S_{A}} A$, that is, $s(x)>0$, for all $x \in \bigcap_{\alpha \in I} A_{\alpha}$, in contradiction with the definition of $K$. The result follows.

THEOREM 2.9. $\partial_{S} X$ is closed if and only if the Choquet topology is separated.

Proof. The family of all sets $\{x \in X: s(x)>0\}\left(s \in S_{1}\right)$ is a base for the topology on $X$ because $X$ is compact. If $\partial_{S} X$ is closed, then it is obvious that the Choquet topology is separated since it coincides with the trace of the topology of $X$ on $\partial_{S} X$. Conversely, if the Choquet topology is separated, then, by virtue of the above two theorems, one obtains that any set $\partial_{S} X \cap\{x \in X: s(x) \leq 0\}(s \in S)$ is closed in this topology and because $\partial_{S} X$ is compact with respect to the Choquet topology it follows that $\partial_{S} X$ is closed.

COROLLARY 2.10. If $X$ is any nonempty, compact, and convex set in an arbitrary Hausdorff locally convex space and $S$ is the convex cone of all real continuous and concave functions on $X$, then $\partial_{S} X$ is closed if and only if the Choquet topology is separated.

We now include some examples of Choquet boundaries accompanied by adjusted details and comments.

EXAMPLE 2.11. If $X$ is any nonempty, compact, and convex subset of every Hausdorff locally convex space and $S=\{f: X \rightarrow \mathbb{R} / f$ is continuous and concave $\}$, then its Choquet boundary with respect to $S$ coincides with the set of all extreme points $x$ of $X$, that is, if $y, z \in X$ and there exists $\alpha \in(0,1)$ with $\alpha y+(1-\alpha) z=x$, then $y=z=x$.

EXAMPLE 2.12. Let $X$ be an arbitrary, compact, convex subset of any Hausdorff locally convex space, and let $C(X)$ be the usual Banach space of all real-valued continuous functions on $X$, endowed with the natural supremum norm and the usual order which generates the cone $S$ of Corollary 2.10. If $T: C(X) \rightarrow C(X)$ is the genuine positive (linear) projection and we denote the range of $T$ by $Y$, that is, $Y=T[C(X)]$, then $T$ is called an Altomare projection [2] if and only if the space of all continuous affine functions 
on $X$ is contained in $Y$ and $f_{t, \alpha} \in Y$ whenever $f \in Y, t \in X$, and $\alpha \in[0,1]$, where $f_{t, \alpha}(x)=f[\alpha x+(1-\alpha) t], x \in X$. In this context, we have

$$
\partial_{Y} X=\{x \in X: T f(x)=f(x), \forall f \in C(X)\},
$$

and geometrically it can be viewed as the union for the corresponding faces of $X$.

Therefore, if one denotes as in $\operatorname{ex}(X)$ the set of all extreme points for $X$, defined in the same sense by the previous example, then

$$
\operatorname{ex}(X) \subseteq \partial_{Y} X \subseteq \operatorname{Frt}(X)
$$

where $\operatorname{Frt}(X)$ represents the usual topological boundary of $X$ and $T f$ is the unique function of $Y$ which coincides with $f$ on $\partial_{Y} X$ for every $f \in C(X)$. With respect to every Altomare projection $T$, any continuous function $\varphi: X \rightarrow[0,1]$, and the probability Radon measure $\mu_{x}$ on $X$ given by

$$
\mu_{x}(f)=\varphi(x) T f(x)+[1-\varphi(x)] f(x) \quad(x \in X, f \in C[X]),
$$

the Lototsky-Schnabl operator is defined by

$$
L_{n, \varphi} f(x)=\int_{X} f\left(\frac{\sum_{i=1}^{n} t_{i}}{n}\right) d \mu_{x}\left(t_{1}\right) \cdots d \mu_{x}\left(t_{n}\right), \quad \forall f \in C(X), x \in X, n \in \mathbb{N}^{*}
$$

Hence, $L_{n, \varphi} Y=y$, for all $y \in Y, L_{n, \varphi} f=f$, for all $f \in \partial_{Y} X$, and $\lim _{n \rightarrow \infty} L_{n, \varphi} f=f$, for all $f \in C(X)$.

EXAMPLE 2.13. In final part of Example 2.12, following [2, 3], we consider the infinitesimal generator $A_{\varphi}: D\left(A_{\varphi}\right) \rightarrow C(X)$ of the attached Feller semigroup $\left(T_{\varphi}(t)\right)_{t \geq 0}$, which coincides with the closure of the operator $W_{\varphi}: D\left(W_{\varphi}\right) \rightarrow C(X)$ defined by

$$
W_{\varphi} f=\lim _{n \rightarrow \infty} n\left(L_{n, \varphi} f-f\right), \quad \forall f \in D\left(W_{\varphi}\right)=\left\{f \in C(X): \exists \lim _{n \rightarrow \infty} n\left(L_{n, \varphi} f-f\right) \text { in } C(X)\right\} .
$$

Then, $\partial_{Y} X=\left\{x \in X: A_{\varphi} f(x)=0, \forall f \in D\left(A_{\varphi}\right)\right\}$ [5] and, from Markov's point of view, it describes the set of all trop points.

Moreover, in all finite $p$-dimensional cases, using [4], one obtains

$$
\partial_{Y} X=\{x \in X: \operatorname{Te}(x)=e(x)\}=\bigcap_{i=1}^{p}\left\{x \in X: T h_{i}^{2}(x)=h_{i}^{2}(x)\right\}
$$

with $T=\lim _{t \rightarrow \infty} T_{\varphi}(t), e=\sum_{i=1}^{p} h_{i}^{2}$, and $h_{i}\left(x_{1}, x_{2}, \ldots, x_{p}\right)=x_{i}$, for all $i=\overline{1, p},\left(x_{1}, x_{2}, \ldots\right.$, $\left.x_{p}\right) \in X$. 
In accordance with [19], if $X=\left\{x \in \mathbb{R}^{p}:\|x\| \leq 1\right\} T: C(X) \rightarrow C(X)$ is the Altomare projection with $T f(f \in C(X))$ being the unique harmonic function on $\operatorname{int}(X)$ which coincides with $f$ on $\operatorname{Frt}(X)$ and $\Delta$ is the Laplace operator, then

$$
A_{\varphi} f(x)=\varphi(x) \frac{1-\|x\|^{2}}{2 p} \Delta f(x), \quad \forall f \in C^{2}(X) .
$$

Consequently,

$$
\partial_{Y} X=\left\{x \in X: \varphi(x) \frac{1-\|x\|^{2}}{2 p} \Delta f(x)=0, \forall f \in C^{2}(X)\right\}
$$

When $X=[0,1], T f(x)=(1-x) f(0)+x f(1)$, for all $x \in[0,1], f \in C([0,1])$, and $\varphi:[0,1] \rightarrow[0,1]$ is any continuous function, then the corresponding Lototsky-Schnable operators are given by

$$
\begin{gathered}
L_{n, \varphi} f(x)=\sum_{k=0}^{n} \sum_{i=0}^{n}\left(\begin{array}{l}
n \\
k
\end{array}\right)\left(\begin{array}{l}
k \\
i
\end{array}\right) \varphi^{k}(x)[1-\varphi(x)]^{n-k} x^{i}(1-x)^{k-i} f\left[\frac{i}{n}+\left(1-\frac{k}{n}\right) x\right], \quad n \in \mathbb{N}^{*}, \\
f \in C(X), \quad A_{\varphi} f(x)=\varphi(x) \frac{x(1-x)}{2} f^{\prime \prime}(x), \\
\forall f \in D\left(A_{\varphi}\right)=\left\{g \in C^{2}([0,1]) \exists \lim _{x \rightarrow 0,1} x(1-x) g^{\prime \prime}(x)=0\right\} .
\end{gathered}
$$

Therefore,

$$
\partial_{Y} X=\left\{x \in X: \frac{\varphi(x) x(1-x)}{2} f^{\prime \prime}(x)=0, \forall f \in D\left(A_{\varphi}\right)\right\} .
$$

3. Efficiency, Choquet boundaries, and related topics. First of all, we recall the Pareto-type efficiency concept in ordered vector spaces and our main results on the coincidence between the sets of the efficient (approximative efficient) point sets and the Choquet boundaries in separated locally convex spaces. In the general context of ordered (topological) vector spaces, let $E$ be an arbitrary vector space ordered by any convex cone $K$. If $A$ is a nonempty arbitrary chosen subset of $E$ and $a_{0} \in A$, then we have the following.

DEFINITION 3.1. $a_{0}$ is said to be a $K$-efficient point of $A$ (a Pareto-type minimum for $A$ with respect to $K$ ), in notation, $a_{0} \in \operatorname{eff}(A, K)$ (or $a_{0} \in \operatorname{MIN}_{K}(A)$ ) if it satisfies one of the following equivalent conditions:

(i) $A \cap\left(a_{0}-K\right) \subseteq a_{0}+K$;

(ii) $K \cap\left(a_{0}-A\right) \subseteq-K$;

(iii) $(A+K) \cap\left(a_{0}-K\right) \subseteq a_{0}+K$;

(iv) $K \cap\left(a_{0}-A-K\right) \subseteq-K$. 
Whenever $K$ is pointed, that is, $K \cap(-K)=\{0\}$, then $a_{0} \in \operatorname{eff}(A, K)$ means that $a_{0}$ fulfills one of the next equivalent relations:

(a) $A \cap\left(a_{0}-K\right)=\left\{a_{0}\right\}$;

(b) $K \cap\left(a_{0}-A\right)=\{0\}$;

(c) $A \cap\left(a_{0}-K \backslash\{0\}\right)=\varnothing$;

(d) $(K \backslash\{0\}) \cap\left(a_{0}-A\right)=\varnothing$.

In a similar manner the Pareto-type maximum elements of $A$ are defined. In fact, $a_{0}^{\prime} \in A$ is a Pareto-type maximum point for $A$ with respect to $K$, in notation, $a_{0}^{\prime} \in$ $\operatorname{MAX}_{K}(A)$, if it is a Pareto minimum point of $A$ with respect to $(-K)$, that is, $a_{0}^{\prime} \in$ $\operatorname{eff}(A,-K)$, that is, $a_{0}^{\prime} \in \operatorname{MIN}_{K}(A)$.

The immediate connection with the fixed points for multifunctions is obviously contained in the following.

REMARK 3.2. $a_{0} \in \operatorname{eff}(A, K)$ if and only if it is a fixed point for at least one of the following multifunctions:

$$
\begin{aligned}
& F_{1}: A \longrightarrow A, \quad F_{1}(t)=\{\alpha \in A: A \cap(\alpha-K) \subseteq t+K\}, \\
& F_{2}: A \longrightarrow A, \quad F_{2}(t)=\{\alpha \in A: A \cap(t-K) \subseteq \alpha+K\}, \\
& F_{4}: A \longrightarrow A, \quad F_{4}(t)=\{\alpha \in A:(A+K) \cap(t-K) \subseteq \alpha+K\},
\end{aligned}
$$

that is, $a_{0} \in F_{i}\left(a_{0}\right)$ for some $i=\overline{1,4}$.

REMARK 3.3. It is known (see [8, 9, 10, 11, 12]) that if $A \subseteq E$ is an arbitrary nonempty set, then a point-to-set mapping $\Gamma: A \rightarrow 2^{A}$ is called a generalized dynamical system when $\Gamma(x) \neq \varnothing$ for every $x \in A$. A point $a_{0} \in A$ is said to be a critical (sometimes equilibrium) point for $\Gamma$ if $\Gamma\left(a_{0}\right)=\left\{a_{0}\right\}$. It is easy to see that whenever $K$ is a pointed, convex cone in $E$, then $a_{0} \in \operatorname{eff}(A, K)$ if and only if $a_{0}$ is a critical point for the generalized dynamical system $\Gamma$ defined by $\Gamma(a)=A \cap(a-K), a \in A$. Thus, one can say that eff $(A, K)$ describes a state of equilibrium for $\Gamma$ and the ideal equilibria contained in this set. In [11], Isac established the following recent general constructive existence principle for $K$-efficient points of nonempty, closed subsets in sequentially complete locally convex spaces, that is, in separated locally convex spaces having the property that every Cauchy sequence is convergent.

THEOREM 3.4. Let ( $\left.E, P=\left\{p_{\alpha}: \alpha \in I\right\}\right)$ be a sequentially complete locally convex space, $K$ a pointed, closed convex cone, $A \subset X$ a nonempty closed subset, and let the generalized dynamical system $\Gamma: A \rightarrow 2^{A}$ be defined by $\Gamma(a)=A \cap(a-K), a \in A$. If, for some $a \in A$, there exists a sequence $\left(a_{n}\right)_{n \in \mathbb{N}}$ such that $a_{n+1} \in \Gamma\left(a_{n}\right)$ for all $n \in \mathbb{N}$ and $\lim _{n \rightarrow \infty} \delta_{\alpha}\left[\Gamma\left(a_{n}\right)\right]=0$ whenever $\alpha \in I$, where $\delta_{\alpha}(B)=\sup \left\{p_{\alpha}(x-y): x, y \in B\right\}$, for every nonempty set $B \subseteq A$ and $\alpha \in I$, then there exists $a_{0} \in \operatorname{eff}(A, K)$ such that $a_{0}-a \in K, \lim _{n \rightarrow \infty} a_{n}=a_{0}$, and $\bigcap_{n \in \mathbb{N}} \Gamma\left(a_{n}\right)=\left\{a_{0}\right\}$.

REMARK 3.5. If the strong minimum point set for $A$ denoted by $S(A, K)=\left\{a_{1} \in A\right.$ : $\left.a-a_{1} \in K, \forall a \in A\right\}$ is nonempty, then $\operatorname{eff}(A, K)=S(A, K)$. 
Indeed, $S(A, K) \subseteq \operatorname{eff}(A, K)$ because if $a_{0} \in S(A, K)$ and $a \in A \cap\left(a_{0}-K\right)$, then $a \in$ $a_{0}+K$.

Assume now that $S(A, K) \neq \varnothing$ and there exists $a_{0} \in \operatorname{eff}(A, K) \backslash S(A, K)$. Then, $a^{*}-a_{0} \notin$ $K$ for some $a^{*} \in A$. Let $\bar{a} \in S(A, K)$. Therefore $a_{0}-\bar{a} \in K \backslash\{0\}$ because if $a_{0}=\bar{a}$, one obtains $a_{0} \in S(A, K)$, in conflict with $a^{*}-a_{0} \notin K$.

Hence $a_{0}-\bar{a} \in K \backslash\{0\}$ and $a_{0} \in \operatorname{eff}(A, K)$ imply that $\bar{a} \in a_{0}+K$, which together with $\bar{a} \in S(A, K)$ leads to $a_{0} \in S(A, K)$, a contradiction.

REMARK 3.6. The largest class $\zeta$ of convex cones ensuring the existence for the efficient points in all nonempty compact subsets of every separated topological vector space was defined in [21] as follows: if $V$ is an arbitrary Hausdorff topological vector space, a convex cone $C$ belongs to $\zeta$ if and only if, for every closed vector subspace $L$ of $V, C \cap L$ is a vector subspace whenever its closure $\overline{C \cap L}$ is a vector subspace. A new direction of study not only for the existence but also for important investigations about Pareto-type efficiency in separated ordered locally convex spaces using completeness instead of compactness was offered by the supernormal (nuclear) cones introduced by Isac in 1981 and published in 1983 [8] (see also [9, 10, 11, 12, 17, 18], etc., for connected bibliography).

Under appropriate conditions, in [23, 24], respectively, it is shown that in every separated topological vector space, the largest class of convex cones ensuring the existence of the efficient points in any bounded and complete subset coincides with the class of cones $K$ satisfying one of the following equivalent conditions:

(i) any bounded increasing net contained in $K$ and in a complete subset of $E$ has a limit;

(ii) any bounded monotone net comprised in a complete subset of $E$ has a limit.

Every supernormal cone has the properties (i), (ii), but there exist convex cones having these properties which are not supernormal. Thus, in the classical Banach spaces $L^{p}([a, b])(p>1)$ the usual positive cone is closed, convex and it has the properties (i), (ii), but it is not supernormal. The same conclusion is valid for the cone of all nonnegative functions in any Orlicz space. Moreover, in related research [23, 24], Truong studied the cones admitting strictly positively functionals and the scalarization of some vector optimization problems.

The first important connection between vector optimization and the potential theory is the next coincidence of the efficient point sets and the Choquet boundaries in separated locally convex spaces, which cannot be obtained as a consequence of the axiomatic potential theory and, following the above results, offers new pertinent properties for the efficient point sets and conversely, through the agency of the convex cone of all real continuous functions on the set for which one looks for the efficient points. Thus, if $X$ is any nonempty, compact subset of $E$ and $K$ is an arbitrary closed, convex, pointed cone, then we have the following.

THEOREM 3.7 [7]. eff $(X, K)$ coincides with the Choquet boundary of $X$ with respect to the convex cone of all real continuous functions which are increasing with respect to order relation $\leq_{K}$. Consequently, the set $\operatorname{eff}(X, K)$ endowed with the trace topology $\tau_{X}$ induced on $X$ by $\tau$ is a Baire space. Moreover, if $X$ is metrizable, then $\operatorname{eff}(X, K)$ is a $G_{\delta}$-set in $\left(X, \tau_{X}\right)$. 
Proof. Since it is known that $\partial_{S} X$ endowed with the trace topology of $X$ is a Baire space (Theorem 2.4) and if, in addition, $X$ is metrizable, then $\partial_{S} X$ is a $G_{\delta}$-subset of $X$ (Theorem 2.6); it is sufficient to prove the first part of theorem.

Let $S_{1}=\left\{f \in C(X): f\right.$ is increasing with respect to $\left.\leq_{K}\right\}$. For this special cone (in general, only for it) we have $\partial_{S_{1}} X=\operatorname{eff}(X, K)$. Indeed, if $x \in \partial_{S_{1}}$ and $x^{\prime} \in X$ such that $x^{\prime} \leq_{K} x$, then $s\left(x^{\prime}\right) \leq s(x)$ for all $s \in S_{1}$, therefore $\varepsilon_{x^{\prime}} \leq_{S_{1}} \varepsilon_{x}$. Because $\varepsilon_{x}$ is minimal with respect to $\leq_{S_{2}}$ one deduces $\varepsilon_{x^{\prime}}=\varepsilon_{x}$, that is, $x^{\prime}=x$. Hence $x \in \operatorname{eff}(X, K)$ and $\partial_{S_{1}} X \subseteq \operatorname{eff}(X, K)$.

For the converse inclusion let $f \in C(X)$ be arbitrary and let $\bar{f}: X \rightarrow \mathbb{R}$ be defined by $\bar{f}(x)=\sup \left\{f\left(x^{\prime}\right): x^{\prime} \in X\right.$ and $\left.x^{\prime} \leq_{K} x\right\}$.

It is obvious that $\bar{f}$ is increasing with respect to $\leq_{K}$ and since, for any $x \in X$, the set $\left\{x^{\prime} \in X: x^{\prime} \leq_{K} x\right\}$ is compact, it follows that there exists $x^{0} \in X, x^{0} \leq_{K} x$, such that $\bar{f}(x)=f\left(x^{0}\right)$. Moreover, $\bar{f}$ is upper semicontinuous. Indeed, let $\left(x_{i}\right)_{i \in I}$ be a generalized sequence in $X$ converging to $x \in X$ and for any $i \in I$ let $x_{i}^{0}$ be an element of $X$ such that $x_{i}^{0} \leq_{K} x_{i}$ and $\bar{f}\left(x_{i}\right)=f\left(x_{i}^{0}\right)$. Without loss of generality, we may suppose that the generalized sequence $\left(x_{i}^{0}\right)_{i \in I}$ is also convergent to $y \in X$. Since $f$ is continuous and the order relation $\leq_{K}$ is closed, we obtain $y=\lim x_{i}^{0} \leq \lim x_{i}=x, \bar{f}(x) \geq f(y)=$ $\lim \bar{f}\left(x_{i}^{0}\right)=\lim \bar{f}\left(x_{i}\right)$ and therefore $\bar{f}$ is upper semicontinuous (see also [14, Appendix, Lemma 4]).

We have $f \leq \bar{f}$ and, moreover, for any function $g: X \rightarrow \mathbb{R}$ which is increasing with respect to $\leq_{K}$ with $f \leq g$, it follows that $\bar{f} \leq g$. Indeed

$\bar{f}(x)=\sup \left\{f\left(x^{\prime}\right): x^{\prime} \in X, x^{\prime} \leq_{K} x\right\} \leq \sup \left\{g\left(x^{\prime}\right): x^{\prime} \in X, x^{\prime} \leq_{K} x\right\}=g(x), \quad \forall x \in X$.

Particularly, for any $s \in S_{1}$ with $s \geq f$, we have $s \geq \bar{f}$ and $Q_{s_{1}} f \geq \bar{f} \geq f$.

On the other hand, by virtue of Nachbin's theorem [14, Appendix, Theorem 3], we deduce $Q_{S_{1}} \bar{f}=\bar{f}$ and, since $f \leq \bar{f}$, we have $\bar{f} \leq Q_{S_{1}} f \leq Q_{S_{1}} \bar{f}=\bar{f}$.

Hence $Q_{s_{1}} f=\bar{f}$ for all $f \in C(X)$ which implies that

$$
\partial_{S_{1}} X=\{x \in X: f(x)=\bar{f}(x), \forall f \in C(X)\} .
$$

Let $x_{0} \in \operatorname{eff}(X, K)$ be arbitrary and $f \in C(X)$.

We have $Q_{S} f\left(x_{0}\right)=\bar{f}\left(x_{0}\right)=\sup \left\{f(x): x \in X\right.$ and $\left.x \leq_{K} x_{0}\right\}=f\left(x_{0}\right)$,

$$
x_{0} \in \partial_{S_{1}} X, \quad \operatorname{eff}(X, K) \subseteq \partial_{S_{1}} X .
$$

Thus, we have proved that

$$
\operatorname{eff}(X, K)=\{x \in X: f(x)=\bar{f}(x), \forall f \in C(X)\}=\partial_{S_{1}} X .
$$

Corollary 3.8. (i) eff $(X, K)=\left\{x \in X: f(x)=\sup \left\{f\left(x^{\prime}\right): x^{\prime} \in X \cap(x-K)\right\} \forall f \in\right.$ $C(X)\}$.

(ii) $\operatorname{eff}(X, K)$ and $\operatorname{eff}(X, K) \cap\{x \in X: s(x) \leq 0\} \quad(s \in S)$ are compact sets with respect to Choquet's topology.

(iii) eff $(X, K)$ is a compact subset of $X$. 
REMARK 3.9. As we have already seen, in Remark 3.6 there exist more general conditions than compactness imposed upon a nonempty set $A$ in a separated locally convex space ordered by a convex cone $K$ ensuring that eff $(X, K) \neq \varnothing$. Perhaps our coincidence result suggests a natural extension of the Choquet boundary at least in these pointed out cases.

REMARK 3.10. In the conditions of Theorem 3.7 we consider on eff $(X, K)$ endowed with the trace topology also denoted by $\tau_{x}$ the following game between two partners $A$ and $B$ : each successively chooses a nonempty set belonging to $\tau_{x}$ such that player $A$ makes the first choice and each player must choose a set in $\tau_{x}$ which should be included into the previously chosen set of the other player.

Let $G_{1}, G_{1}^{\prime}, G_{2}, G_{2}^{\prime}, \ldots, G_{n}, G_{n}^{\prime}, \ldots$ be the successive options of the two players; $\left(G_{1}, G_{2}, \ldots, G_{n}, \ldots\right)$ represent the options expressed by $A$ and $\left(G_{1}^{\prime}, G_{2}^{\prime}, \ldots, G_{n}^{\prime}, \ldots\right)$ are the options made by $B$. One says player $B$ wins if, regardless of the way $A$ plays, $B$ is able to make an option so that

$$
\bigcap_{n \in \mathbb{N}^{*}} G_{n} \neq \varnothing
$$

Theorem 3.7 together with Choquet's results (see, e.g., the references corresponding to [6], Chapter 2) concerning the properties of the Choquet boundary shows that the above game on eff $(X, K)$ is won by player $B$. Whenever the above game is won by player $B$, it follows that $X$ is a Baire space. Moreover, the same game on the Choquet boundary of every nonempty compact subset in any Hausdorff locally convex space, with respect to every convex cone of real-valued continuous functions, which contains at least one strictly positive function and separates linearly the basic compact space, is won by the player $B$.

REMARK 3.11. As we have seen in [1], under the hypothesis of Theorem $3.7 \operatorname{eff}(X, K)$ coincides with the Choquet boundary of $X$ only with respect to the convex cone of all real, continuous, and $K$-increasing functions in $X$. Thus, for example, if $X$ is a nonempty, compact, and convex subset of $E$, then, taking into account Example 2.11, the Choquet boundary of $X$ with respect to the convex cone of all real, continuous, and concave functions on $X$ coincides with the set of all extreme points for $A$. But it is easy to see that even in finite-dimensional cases an extreme point for a compact convex set is not necessarily an efficient point and conversely. In order to give the second coincidence result between the approximate efficient point sets and some Choquet boundaries, we consider in the same framework of this section before Theorem 3.7 an arbitrary element $\varepsilon \in K \backslash\{0\}$. Then, it is obvious that the set $G_{\varepsilon-K}=\{(x, y) \in E \times E: y \in x+\varepsilon+K\}$ is closed in $E \times E$ endowed with the usual product topology.

DEFINITION 3.12. If $X$ is a nonempty subset of $E$, then $a_{0} \in A$ will be called a minimal element ( $\varepsilon$-efficient point, Pareto $\varepsilon$-efficient point, $\varepsilon$-near-to-minimum point) of $A$ with respect to $K$ if there exists no $a \in A$ such that $a_{0}-a-\varepsilon \in K$, that is, $\left(a_{0}-\varepsilon-K\right) \cap A=\varnothing$.

The $\varepsilon$-efficient point set of $A$ with respect to $K$ will be denoted by $\varepsilon-\operatorname{eff}(A, K)$ (or $\varepsilon-\operatorname{MIN}_{K}(A)$ ). Some approximate solutions of vector optimization problems and 
connected generalizations for Pareto-type optimums are given, for example, in [13, 20], respectively.

REMARK 3.13. It is clear that the concept of the $\varepsilon$-efficient point does not include the notion of efficient point, $\operatorname{eff}(A, K) \subseteq \varepsilon-\operatorname{eff}(A, K)$, for all $\varepsilon \in K \backslash\{0\}$, and $\operatorname{eff}(A, K)=$ $\bigcap_{\varepsilon \in K \backslash\{0\}}[\varepsilon-\operatorname{eff}(A, K)]$.

REMARK 3.14. A very interesting and important generalization of the approximative Pareto efficiency given by Definition 3.12 was considered in [15] by replacing $\varepsilon$ with a nonempty subset of $K \backslash\{0\}$. In this way it is shown that the existence of this new type of efficient point set for lower-bounded sets characterizes the semi-Archimedian ordered vector spaces and the regular ordered locally convex spaces. In [15], we also find many pertinent examples and comments.

DEFINITION 3.15. A real function $f: E \rightarrow \mathbb{R}$ is called $(\varepsilon+K)$-increasing if $f\left(x_{1}\right) \geq$ $f\left(x_{2}\right)$ whenever $x_{1}, x_{2} \in E$ and $x_{1} \in x_{2}+\varepsilon+K$.

THEOREM 3.16. If $X$ is any nonempty and compact subset of $E$, then the set $\varepsilon-$ eff $(X, K)$ coincides with the Choquet boundary of $X$ with respect to the convex cone and all $(\varepsilon+K)$-increasing real continuous functions on $X$. Consequently, the set $\varepsilon-\operatorname{eff}(x, K)$ endowed with the trace topology is a Baire space and if $\left(X, \tau_{x}\right)$ is metrizable, then $\varepsilon-\operatorname{eff}(x, K)$ is a $G_{\delta}$-subset of $X$.

Proof. The proof follows approximatively the same line as in Theorem 3.7. Thus, Theorems 2.4 and 2.6 show that the last part of the above theorem is a consequence of the usual properties for Choquet boundaries under the specified conditions. Let $S_{1}=\{f \in C(X): f$ is $(\varepsilon+K)$-increasing $\}$. Clearly, $S_{1}$ is a convex cone which contains the constant functions on $X$, it is min-stable, and it separates the points of $X$. If $x^{*} \in \partial_{S_{1}} X$ and $x^{\prime} \in X$ with $x^{*} \in x^{\prime}+\varepsilon+K$, then $s\left(x^{*}\right) \geq s\left(x^{\prime}\right)$ for all $s \in S_{1}$, therefore $\varepsilon_{X^{\prime}} \leq \varepsilon_{X^{*}}$. Because $\varepsilon_{x^{*}}$ is minimal with respect to " $\leq_{S_{1}}$ " one deduces $\varepsilon_{x^{*}}=\varepsilon_{x^{\prime}}$, that is, $x^{*}=x^{\prime}$ (if $x^{*} \neq x^{\prime}$, then, from the fact that $S_{1}$ separates the points of $X$, it follows that there exists $f \in S_{1}$ with $f\left(x^{*}\right) \neq f\left(x^{\prime}\right)$, in conflict with the equality $\varepsilon_{x^{*}}=\varepsilon_{x^{\prime}}$, which means that $f\left(x^{*}\right)=f\left(x^{\prime}\right)$, for all $\left.f \in C(X)\right)$; or $0 \notin \varepsilon+K$, so $x^{*}=x^{\prime}$ is a contradiction and this proves the inclusion $\partial_{S_{1}} X \subseteq \varepsilon-\operatorname{eff}(X, K)$. For the converse inclusion let $f \in C(X)$ be arbitrary and let $\bar{f}: X \rightarrow \mathbb{R}$ be defined by $\bar{f}(x)=\sup \left\{f\left(x^{\prime}\right): x^{\prime}=x\right.$ or $x^{\prime} \in X$ and $x \in$ $\left.x^{\prime}+\varepsilon+K\right\}$. It is obvious that $\bar{f}$ is $(\varepsilon+K)$-increasing and since for any $x \in X$ the set $\left\{x^{\prime} \in X: x^{\prime}=x\right.$ or $x^{\prime} \in X$ and $\left.x \in x^{\prime}+\varepsilon+K\right\}$ is compact, it follows that there exists $x_{0} \in X$ with $x \in x_{0}+\varepsilon+K$ such that $\bar{f}(x)=f\left(x_{0}\right)$. Moreover, $\bar{f}$ is upper semicontinuous (see also, e.g., [14, Appendix, Lemma 4]). We have $f \leq \bar{f}$ and for any function $g \in S_{1}$ with $f \leq g$ it follows that $\bar{f} \leq g$ because

$$
\begin{aligned}
\bar{f}(x) & =\sup \left\{f\left(x^{\prime}\right): x^{\prime}=x \text { or } x^{\prime} \in X, x \in x^{\prime}+\varepsilon+K\right\} \\
& \leq \sup \left\{g\left(x^{\prime}\right): x^{\prime}=x \text { or } x^{\prime} \in x+\varepsilon+K, x \in x^{\prime}+\varepsilon+K\right\} \\
& =g(x), \quad \forall x \in X .
\end{aligned}
$$

Particularly, for any $s \in S_{1}$ with $s \geq f$ we have $s>\bar{f} Q_{S_{1}} f \geq \bar{f} \geq f$. On the other hand, by virtue of Nachbin's theorem [14, Appendix, Theorem 3] one deduces $Q_{S_{1}} \bar{f}=\bar{f}$. Since 
$f \leq \bar{f}$, we have $\bar{f} \leq Q_{S_{1}} f \leq Q_{S_{1}} \bar{f}=\bar{f}$. Hence $Q_{S_{1}} f=\bar{f}$, for all $f \in C(X)$, which implies that $\partial_{S_{1}} X=\{x \in X: f(x)=\bar{f}(x), \forall f \in C(X)\}$. Let $x_{0} \in \varepsilon-\operatorname{eff}(X, K)$ and $f \in C(X)$. We have

$$
Q_{S_{1}} f\left(x_{0}\right)=\bar{f}\left(x_{0}\right)=\sup \left\{f(x): x=x_{0} \text { or } x \in X, x_{0} \in x+\varepsilon+K\right\}=f\left(x_{0}\right),
$$

therefore $x_{0} \in \partial_{S_{1}} X$ and $\varepsilon-\operatorname{eff}(X, K) \subseteq \partial_{S_{1}} X$. Thus we have proved that

$$
\varepsilon-\operatorname{eff}(X, K)=\{x \in X: f(x)=\bar{f}(x), \forall f \in C(X)\}=\partial_{S_{1}} X .
$$

Now, let $D$ be a nonempty subset of $E$, let $k^{0} \in E$, and let $\varepsilon>0$ be such that $D+\alpha k^{0} \subseteq$ $D$ for all $\alpha>0$. If one denotes $D_{\varepsilon k^{0}}=\varepsilon k^{0}+(D \backslash\{0\})$ and we consider a proper subset $A$ of $E$, then we have the following.

Definition 3.17 [22]. An element $x_{0} \in A$ is called $\varepsilon k^{0}$-efficient of $A$ with respect to $D$ if there is no $x \in A$ with $x_{\varepsilon} \in x+D_{\varepsilon k^{0}}$.

In accordance with [22], the $\varepsilon k^{0}$-efficient of $A$ with respect to $D$ will be denoted by $\operatorname{eff}\left(A, D_{\varepsilon k^{0}}\right)$.

DEFINITION 3.18 [22]. We say that a function $f: E \rightarrow \mathbb{R}$ is $D_{\varepsilon k^{0}}$-increasing if $x_{1} \in$ $x_{2}+D_{\varepsilon k^{0}}$ implies that $f\left(x_{1}\right) \geq f\left(x_{2}\right)$.

REMARK 3.19. Following the above theorem, it is clear that if $X$ is a nonempty, compact subset of $E$ and $\partial_{S} X$ denotes its corresponding Choquet boundary with respect to the convex cone

$$
S=\left\{f \in C(X): f \text { is } D_{\varepsilon k^{0}} \text {-increasing }\right\},
$$

then $\partial_{S} X \subseteq \operatorname{eff}\left(X, D_{\varepsilon k^{0}}\right)$

The second part of the proof given for Theorem 3.16 shows that except for some particular cases, it seems that the condition for $D$ to be a proper, closed, convex, and pointed cone is essential in order to have equality in this last inclusion relation.

REMARK 3.20. In our opinion, the immediate intrinsic topological study of Choquet's boundaries with respect to the specific (Choquet) topologies and the special refined facial topologies specified also in the fourth paragraph of the second chapter in [6] is important for the investigations on the sensitivity of the corresponding efficient and approximate efficient point sets, thanks to the above coincidence results between the (approximate) efficient point sets and the Choquet boundaries.

4. Some open problems. The above research suggests immediately the following open problems.

Problem 4.1. If eff $(A, K) \neq \varnothing$, there exist a Hausdorff locally convex space $Y$, a supernormal cone $K_{0}$ in $Y$, and a nonempty set $A_{0} \subset Y$ with $\operatorname{eff}(A, K)=\operatorname{eff}\left(A_{0}, K_{0}\right)$ (or, at least, eff $(A, K)$ is dense in $\left.\operatorname{eff}\left(A_{0}, K_{0}\right)\right)$. 
Problem 4.2. If eff $(A, K) \neq \varnothing$, there exist a separated locally convex space $X_{1}$, a (pointed) convex cone $K_{1}$ in $X_{1}$, and a compact set $A_{1} \subset X_{1}$ such that $\operatorname{eff}(A, K)=$ $\operatorname{eff}\left(A_{1}, K_{1}\right)$ (or, at least, eff $(A, K)$ to be dense in $\left.\operatorname{eff}\left(A_{1}, K_{1}\right)\right)$.

Problem 4.3. If $E$ is a Hilbert space, $K$ is a closed, convex, pointed cone in $E$, and $X$ is a nonempty, closed, convex subset of $E$, does eff $(X, K)$ preserve the property of coincidence with the corresponding Choquet boundary as in the above theorem?

Problem 4.4. The same question applies in each of the following cases:

(i) $E$ is any quasicomplete locally convex space, $K$ denotes every supernormal ordering cone for $E$, and $X$ is an arbitrary $K$-bounded and $K$-closed subset of $E$ [12];

(ii) $E$ represents every quasicomplete locally convex space, the closure $\bar{K}$ of an arbitrary ordering cone $K$ in $E$ has one of the equivalent properties (i) or (ii) given in Remark 3.6, and $X$ is any $K$-bounded and $K$-closed subset in $E$.

ACKNOWLEDGMENT. The author would like to thank the Managing Editor for valuable suggestions which improved this research work.

\section{REFERENCES}

[1] E. M. Alfsen, Compact Convex Sets and Boundary Integrals, Springer-Verlag, New York, 1971.

[2] F. Altomare, Limit semigroups of Bernstein-Schnabl operators associated with positive projections, Ann. Scuola Norm. Sup. Pisa Cl. Sci. (4) 16 (1989), no. 2, 259-279.

[3] F. Altomare and M. Campiti, Korovkin-Type Approximation Theory and Its Applications, de Gruyter Studies in Mathematics, vol. 17, Walter de Gruyter, Berlin, 1994.

[4] F. Altomare and I. Rasa, Towards a characterization of a class of differential operators associated with positive projections, Atti Sem. Mat. Fis. Univ. Modena 46 (1998), no. suppl., 3-38.

[5]___ Feller semigroups, Bernstein type operators and generalized convexity associated with positive projections, New Developments in Approximation Theory (Dortmund, 1998), Internat. Ser. Numer. Math., vol. 132, Birkhäuser, Basel, 1999, pp. 9-32.

[6] N. Boboc and Gh. Bucur, Conuri Convexe de Funcții Continue pe Spații Compacte [Convex Cones of Continuous Functions on Compact Spaces], Editura Academiei Republicii Socialiste România, Bucharest, 1976.

[7] I. Bucur and V. Postolică, A coincidence result between sets of efficient points and Choquet boundaries in separated locally convex spaces, Optimization 36 (1996), no. 3, 231234.

[8] G. Isac, Sur l'existence de l'optimum de Pareto, Riv. Mat. Univ. Parma (4) 9 (1983), 303-325.

[9] _ Supernormal cones and absolute summability, Libertas Math. 5 (1985), 17-32.

[10] __ Pareto optimization in infinite-dimensional spaces: the importance of nuclear cones, J. Math. Anal. Appl. 182 (1994), no. 2, 393-404.

[11] _ On Pareto efficiency. A general constructive existence principle, Combinatorial and Global Optimization (Chania, 1998) (P. M. Pardalos, A. Migdalas, and R. Burkard, eds.), Ser. Appl. Math., vol. 14, World Scientific Publishing, New Jersey, 2002, pp. 133144.

[12] G. Isac and V. Postolică, The Best Approximation and Optimization in Locally Convex Spaces, Approximation \& Optimization, vol. 2, Peter Lang, Frankfurt am Main, 1993.

[13] P. Loridan, $\epsilon$-solutions in vector minimization problems, J. Optim. Theory Appl. 43 (1984), no. 2, 265-276.

[14] L. Nachbin, Topology and Order, D. Van Nostrand, New Jersey, 1965. 
[15] A. B. Németh, Between Pareto efficiency and Pareto $\epsilon$-efficiency, Optimization 20 (1989), no. $5,615-637$.

[16] A. L. Peressini, Ordered Topological Vector Spaces, Harper \& Row Publishers, New York, 1967.

[17] V. Postolică, New existence results for efficient points in locally convex spaces ordered by supernormal cones, J. Global Optim. 3 (1993), no. 2, 233-242.

[18] _ Properties of Pareto sets in locally convex spaces, Optimization 34 (1995), no. 3, 223-229.

[19] I. Rasa, Feller semigroups, elliptic operators and Altomare projections, Rend. Circ. Mat. Palermo (2) Suppl. (2002), no. 68, 133-155.

[20] T. Staib, On two generalizations of Pareto minimality, J. Optim. Theory Appl. 59 (1988), no. 2, 289-306.

[21] A. Sterna-Karwat, On existence of cone-maximal points in real topological linear spaces, Israel J. Math. 54 (1986), no. 1, 33-41.

[22] Chr. Tammer, A generalization of Ekeland's variational principle, Optimization 25 (1992), no. 2-3, 129-141.

[23] X. D. H. Trúóng, A note on a class of cones ensuring the existence of efficient points in bounded complete sets, Optimization 31 (1994), no. 2, 141-152.

[24] On the existence of efficient points in locally convex spaces, J. Global Optim. 4 (1994), no. 3, 265-278.

Vasile Postolică: Faculty of Sciences, Romanian Academy of Scientists - Bacău State University, Bacău 600115, Romania

E-mail address: vpostolica@ambra.ro; vpostolica@ub.ro 


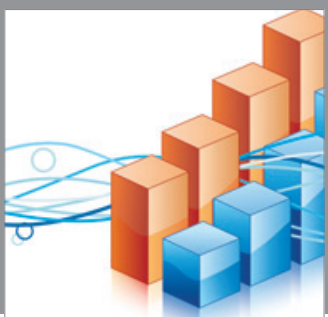

Advances in

Operations Research

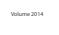

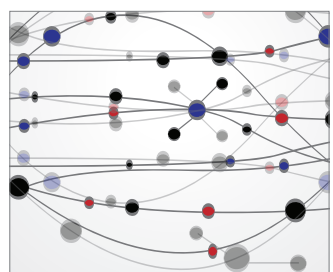

\section{The Scientific} World Journal
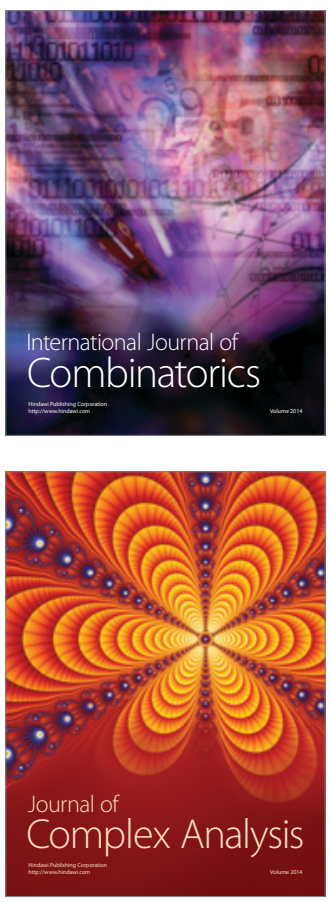

International Journal of

Mathematics and

Mathematical

Sciences
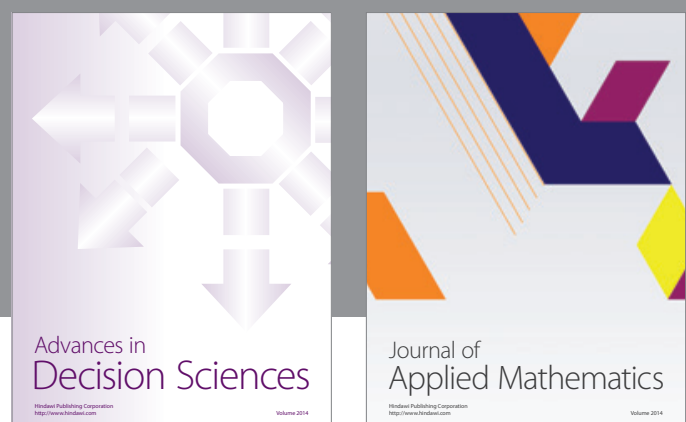

Journal of

Applied Mathematics
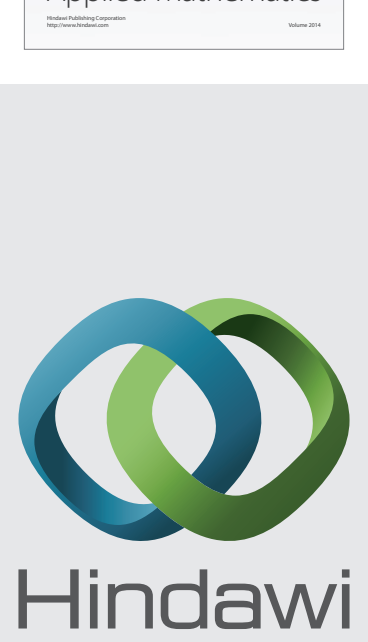

Submit your manuscripts at http://www.hindawi.com
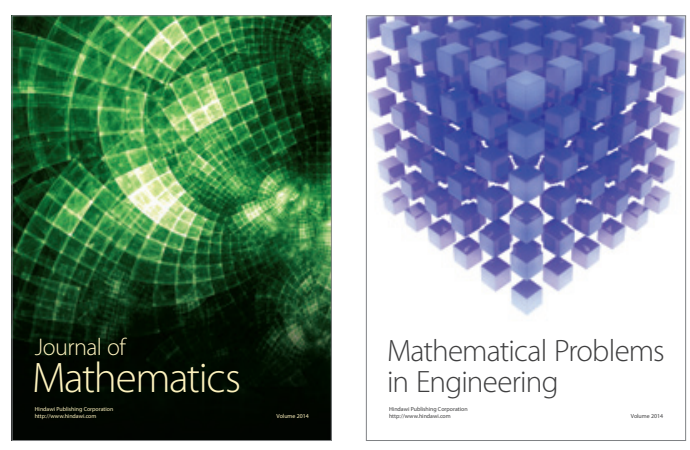

Mathematical Problems in Engineering
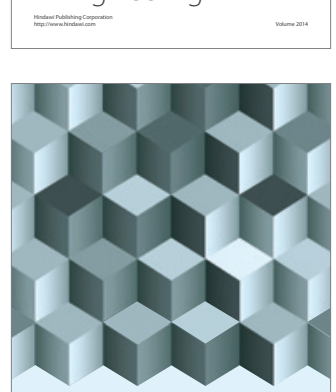

Journal of

Function Spaces
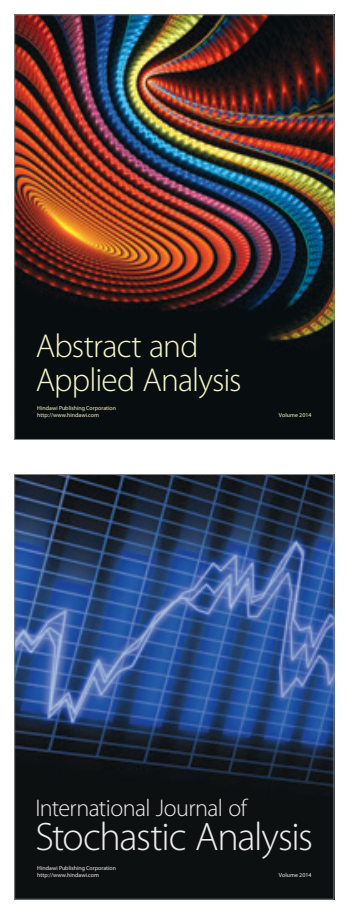

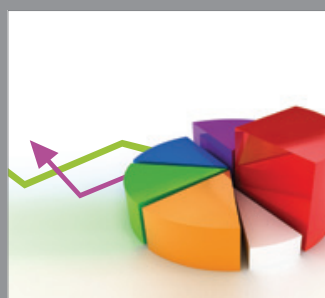

ournal of

Probability and Statistics

Promensencen
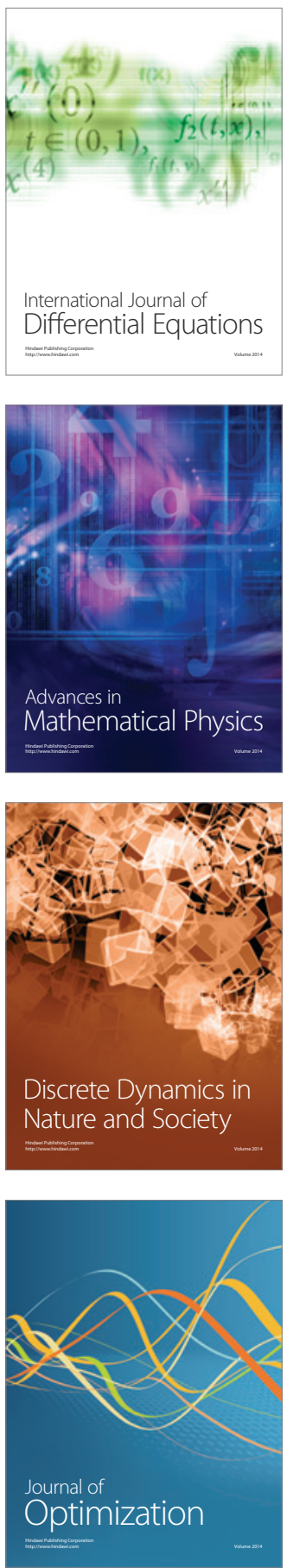